Research Institute for Advanced Computer Science NASA Ames Research Center

\title{
New Computational Methods for the Prediction and Analysis of Helicopter Noise
}

\author{
Roger C. Strawn \\ Leonid Oliker \\ Rupak Biswas
}





\title{
New Computational Methods for the Prediction and Analysis of Helicopter Noise
}

\author{
Roger C. Strawn \\ Leonid Oliker \\ Rupak Biswas
}

The Research Institute of Advanced Computer Science is operated by Universities Space Research Association, The American City Building, Suite 212, Columbia, MD 21044, (410) 730-2656

Work reported herein was supported by NASA via Contract NAS 2-13721 between NASA and the Universities Space Research Association (USRA). Work was performed at the Research Institute for Advanced Computer Science (RIACS), NASA Ames Research Center, Moffett Field, CA 94035-1000. 



\title{
NEW COMPUTATIONAL METHODS FOR THE PREDICTION AND ANALYSIS OF HELICOPTER NOISE*
}

\author{
Roger C. Strawn ${ }^{\dagger}$ \\ US Army AFDD, M/S 258-1, NASA Ames Research Center, Moffett Field, CA 94035 \\ Leonid Oliker ${ }^{\ddagger}$ \\ RIACS, M/S T27A-1, NASA Ames Research Center, Moffett Field, CA 94035 \\ Rupak Biswas ${ }^{\S}$ \\ RIACS, M/S T27A-1, NASA Ames Research Center, Moffett Field, CA 94035
}

\begin{abstract}
This paper describes several new methods to predict and analyze rotorcraft noise. These methods are: 1) a combined computational fluid dynamics and Kirchhoff scheme for far-field noise predictions, 2) parallel computer implementation of the Kirchhoff integrations, 3 ) audio and visual rendering of the computed acoustic predictions over large far-field regions, and 4) acoustic tracebacks to the Kirchhoff surface to pinpoint the sources of the rotor noise. The paper describes each method and presents sample results for three test cases. The first case consists of in-plane high-speed impulsive noise and the other two cases show idealized parallel and oblique blade-vortex interactions. The computed results show good agreement with available experimental data but convey much more information about the far-field noise propagation. When taken together, these new analysis methods exploit the power of new computer technologies and offer the potential to significantly improve our prediction and understanding of rotorcraft noise.
\end{abstract}

\section{INTRODUCTION}

Future helicopter and tiltrotor designs must have low noise if they are to operate successfully near heavily populated areas. This requires the reduction of two main types of aerodynamic noise from the rotor system. The first is called high-speed impulsive (HSI) noise and is characterized by a strong acoustic disturbance occurring over a very short period of time. Impulsive noise is generally associated with high tip speeds and advancing-tip Mach numbers greater than 0.9 . The second type of noise comes from the interaction of the rotor

*Presented at the 2nd AIAA/CEAS Aeroscoustics Conference, 6-8 May, 1996, State College, PA. This paper is declared a work of the U.S. Government and is not subject to copyright protection in the United States

${ }^{\dagger}$ Research Scientist, Member AIAA.

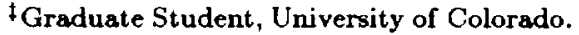

SScientist, Member AIAA. blades with their vortical wake systems. These bladevortex interactions (BVI's) are particularly important near urban areas when helicopters are descending for landings.

Rotorcraft designers require three things in order to systematically reduce HSI and BVI noise. First, they need high-accuracy methods for predicting far-field rotor noise. Second, they need a way to analyze these predictions that identifies the causes of the offending noise. The third requirement is an ability to alter the rotor blade motion to reduce the far-field noise. This paper describes several new acoustics prediction and analysis methods that address the first two of these objectives. These new methods focus heavily on new and emerging computer algorithms, audio and visual rendering tools, and parallel processing. These new technologies provide a powerful framework for improved understanding of rotorcraft noise with an aim to reducing it.

Recent advances in rotorcraft noise prediction have come from combinations of computational fluid dynamics (CFD) and Kirchhoff methods. Finite-difference solutions to the Full Potential or Euler/Navier-Stokes equations are used close to the rotor blades to model the near-field aerodynamics and acoustics. These nearfield solutions are then interpolated onto a surface that completely encloses the rotor blades. A Kirchhoff integration over this surface carries the near-field acoustics solution to any point in the far field. These hybrid methods have the advantage that the nonlinear acoustic propagation is accurately modeled by the CFD solution close to the rotor blades where compressibility is important. Away from the rotor blades, it is no longer practical to compute the CFD solution over a large volume because numerical dissipation causes a loss of accuracy in the acoustic signal. The Kirchhoff integration is much more computationally efficient than the CFD methods for acoustic propagation over large distances with no dissipation. It assumes a constant speed of sound outside the Kirchhoff surface, which is a very good approximation away from the rotor blades. 
Examples of these hybrid CFD/Kirchhoff methods for computing both HSI and BVI helicopter noise are given in Refs. [1-11].

Up until now, these CFD/Kirchhoff techniques have been used to compute acoustic signals at a handful of far-field observer locations in order to compare with experimental microphone measurements. While these types of comparisons are useful for the purpose of validation, they do not exploit the full capabilities of these new acoustic prediction methods. The CFD/Kirchhoff formulations can compute far-field acoustic pressures at many observer locations covering large regions of the flowfield. When viewed as a whole, these acoustic signals give a great deal of insight into the far-field propagation characteristics of helicopter noise. This is particularly important for tiltrotor BVIs where many of the noise generation and propagation mechanisms are not fully understood. Recent papers by Lowson [12] and Sim et al. [13] clearly emphasize the need for detailed studies of far-field helicopter noise propagation.

This paper describes several new computational tools for computing and analyzing helicopter noise in the far field using the new CFD/Kirchhoff formulation. First, we present a method for speeding up the Kirchhoff integrations so that several thousand acoustic signals can be computed in a few hours. We accomplish this by running the Kirchhoff code on an IBM SP2 multiprocessor. The Kirchhoff integration is very well suited for parallel implementation and gives nearideal speedups when modified to run on the SP2. Once the Kirchhoff code has computed the acoustic signals for all the far-field observer locations, these signals are postprocessed to obtain visual and auditory information about the resulting noise. A computer graphics program creates animated three-dimensional images of the acoustic pressure waves while an interactive audio system converts the computed digital acoustic signals into sound. In addition, we present a new technique to identify the sources of far-field noise on the Kirchhoff surface. We demonstrate these new analysis tools for rotorcraft cases with both HSI and BVI noise.

\section{CFD/KIRCHHOFF INTEGRATION METHOD}

The CFD/Kirchhoff method consists of a CFD solution near the rotor blades followed by a Kirchhoff integration to propagate the acoustic signals to the far field. In this paper, an Euler/Navier-Stokes flow solver called TURNS $[14,15]$ computes the near-blade CFD solution. The TURNS code solves the Navier-Stokes equations about rotating helicopter blades. Since viscous effects are small for the cases considered in this paper, we run the TURNS code in the Euler mode. All nonlinear effects on the acoustic propagation are accurately modeled within the framework of the Euler equations.

The Kirchhoff formulation from Farassat and Myers [16] is used to evaluate the acoustic pressure, $P$, at a fixed observer location, $\vec{x}$, and observer time, $t$. In the general case, the Kirchhoff surface, $S$, can deform and have arbitrary motion; however, the applications in this paper use a nonrotational, rigid surface, with linear translational motion. With these simplifications, the Kirchhoff formula becomes:

$$
P(\vec{x}, t)=\frac{1}{4 \pi} \int_{S}\left[\frac{E_{1}}{|\vec{r}|\left(1-M_{r}\right)}+\frac{E_{2} P}{r^{2}\left(1-M_{r}\right)}\right]_{\tau} \mathrm{d} S .
$$

The expressions for $E_{1}$ and $E_{2}$ are given as:

$$
\begin{gathered}
E_{1}=\left(M_{n}^{2}-1\right) P_{n}+M_{n} \vec{M}_{t} \cdot \nabla_{2} P-\frac{M_{n} \dot{P}}{a_{\infty}}+ \\
\frac{\left(\cos \theta-M_{n}\right) \dot{P}}{a_{\infty}\left(1-M_{r}\right)} \\
E_{2}=\frac{\left(1-M^{2}\right)\left(\cos \theta-M_{n}\right)}{\left(1-M_{r}\right)^{2}} .
\end{gathered}
$$

The Kirchhoff surface translates with the rotor hub at Mach number $\vec{M}$. The distance between a point on the Kirchhoff surface and the observer is given by $|\vec{r}|$. $M_{n}$ and $M_{r}$ are the components of $\vec{M}$ along the local surface normal, $\vec{n}$, and the radiation direction, $\vec{r}, \vec{M}_{t}$ is the Mach number tangent to the Kirchhoff surface, $P_{n}$ is the derivative of $P$ along the surface normal, $\dot{P}$ is the time derivative of pressure, and $\nabla_{2} P$ is the gradient of the pressure on the Kirchhoff surface. The freestream speed of sound is assumed uniform at $a_{\infty}$, and $\theta$ is the angle between $\vec{n}$ and $\vec{r}$. The simplified form for $E_{2}$ in Eq. (3) is taken from Myers and Hausmann [17].

Figure 1 shows a representative Kirchhoff surface in the nonrotating reference frame. The rotor blades spin inside the surface which consists of a top, bottom, and side mesh. Each of these three meshes is discretized with approximately 40,000 points. Evaluation of the acoustic pressure in Eq. (1) first requires a spatial interpolation of pressure and pressure derivatives from the rotating CFD grid onto the nonrotating Kirchhoff mesh. These spatial interpolations are performed in the CFD code at run time. The Kirchhoff surface pressures and pressure derivatives are stored on secondary disk at typical intervals of one degree azimuthal angle for a complete cycle of blade rotation. Overset grid techniques are used for the spatial interpolations onto the Kirchhoff surface. Either the DCF3D code from Meakin [18], or the PEGSUS code from Suhs and Tramel [19] can be used for these interpolations with negligible impact on run time for the CFD codes. References $[7,11]$ give details on the implementation of these interpolation techniques. 


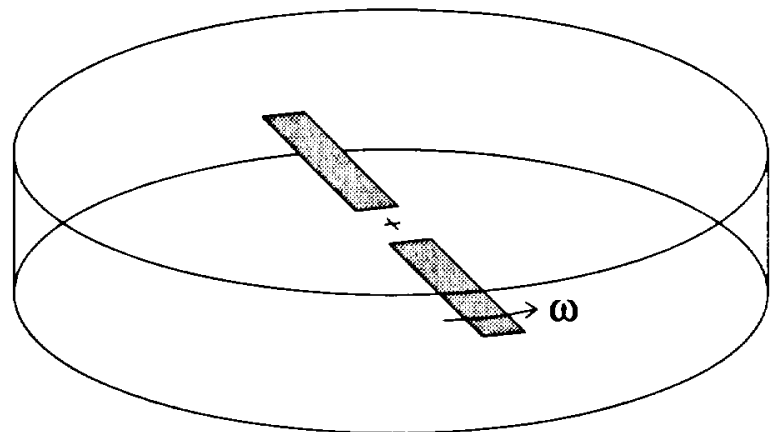

Figure 1: The helicopter blades rotate inside the nonrotating Kirchhoff surface.

Evaluation of the acoustic pressure in Eqs. (1-3) also requires a temporal interpolation in the stored database of Kirchhoff-surface pressures. The integrand must be evaluated at the time of emission, $\tau$, for each differential area on the Kirchhoff surface. This is also known as the retarded time. Appropriate values of retarded time are determined by noting that the time delay between the emission of the acoustic signal and its arrival at the observer is equal to the distance that this signal must travel divided by the freestream speed of sound. This formulation leads to a quadratic equation for $\tau$. One of the roots is non-physical and discarded. We retrieve the appropriate pressure and pressure derivatives at this emission time using linear temporal interpolation in the database of stored pressure values on the Kirchhoff surface.

For a Kirchhoff surface with 129,600 points, the Kirchhoff integration code requires 0.075 Cray C-90 $\mathrm{CPU}$ seconds for each pressure evaluation in Eqs. (13 ), at an observer location, $\vec{x}$, in space, and an observer time, $t$. The Kirchhoff program runs at 470 MFLOPS on the C-90 with improved coding for the interpolations giving an overall speed that is approximately 20 times faster than the CPU times reported in Ref. [7].

An alternative to the nonrotating Kirchhoff formulation described above is to use a Kirchhoff surface that rotates with the rotor blades. The advantage of this rotating-surface formulation is that spatial interpolations onto the Kirchhoff surface are not required since the Kirchhoff grid coincides with part of the rotating CFD mesh. Reference [7] presents results from both the rotating and nonrotating-surface Kirchhoff methods. The two methods used the same CFD solutions as inputs and gave similar results. However, the nonrotating-surface method was more robust, particularly for high-speed rotor cases.

The major drawback for the rotating-surface Kirchhoff method is that the Kirchhoff surface must move subsonically. This requirement places a restriction on the outer radial location for the rotating sur- face. If it is too far from the rotor blade tip, it will have supersonic motion, which is not allowed. On the other hand, if the surface is too close, then the acoustic solution may be inaccurate. The nonrotating-surface formulation does not have this problem since the translating Kirchhoff surface always moves subsonically.

The nonrotating Kirchhoff formulation has several other advantages over its rotating-surface counterpart. First, the computation time for the nonrotating-surface method is independent of the number of rotor blades. The rotating-surface computation time increases proportionately with the number of rotor blades since the Kirchhoff integrations must be performed for each blade to obtain the complete far-field pressure signal. Second, the retarded time equation for the rotating-surface method is transcendental, and requires an iterative solution for each differential element on the Kirchhoff surface at each far-field observer time. As mentioned earlier, the retarded time equation for the nonrotatingsurface method is quadratic and does not require an iterative solution. This difference is most significant on vector computers, such as the C-90, where the iterative retarded time equation is difficult to vectorize. A final advantage of the nonrotating Kirchhoff method is that it can be used to help pinpoint the locations of noise sources in the plane of the rotor. This information is useful for low-noise rotor design and is discussed in detail later in this paper.

\section{PARALLEL IMPLEMENTATION}

Implementation of the rotating and nonrotating Kirchhoff integrations on parallel computers is straightforward due to the regular nature of the computations. The Kirchhoff surface is divided into patches and each patch is assigned to a processor. Virtually no communication is needed among processors except for a final summation of the integral contributions from each surface patch. The Kirchhoff integrations were run on the 148-node IBM SP2 multiprocessor installed at NASA Ames Research Center. The parallel code uses a message-passing paradigm and is portable to any distributed-memory architecture that supports FORTRAN 90 and the MPI library. The parallel implementation strategy is described below.

The Kirchhoff surface and associated pressure files are stored as two dimensional arrays of size (imax,jmax), which must be partitioned and distributed among the processors. Since our SP2 is restricted to serial $\mathrm{I} / \mathrm{O}$, one "host" processor is designated to read and scatter the data to the working set, $P$, of processors. Each processor receives a contiguous one-dimensional strip of $($ imax $\times j \max ) / P$ data elements, plus an additional overlapped section of $i$ max elements. This allows local, communication-free, nearent- 
neighbor computations. Note that the data could have also been distributed in two-dimensional blocks of size ( $i \max / P, j \max / P$ ), thereby reducing the space required for the overlapped elements. However, this would adversely affect cache performance and complicate the local index calculations. Due to the relatively small value of $i \max$, the former less complex approach was taken.

Since no communication is required until a final global summation, and the $I / O$ is independent of the number of processors, the actual run time on the SP2 scales by over $98 \%$ as the number of processors increases. As a result, with 60 processors on the SP2, and 86,400 points on the Kirchhoff surface, we can compute a periodic time history of 180 pressure evaluations for each of 7533 far-field observer locations in about five CPU hours. A similar calculation on one processor of a $\mathrm{C}-90$ requires over twenty CPU hours. The real payoff however, comes from the scalability of the parallel implementation. This same calculation would require only about $35 \mathrm{CPU}$ minutes to complete on a 512-node SP2.

\section{VISUAL POSTPROCESSING}

Figure 2 shows a sample grid of far-field observer locations for the OLS rotor blade that was experimentally-tested by Schmitz et al. [20]. These grid points are located in the plane of the rotor between 2 and 12 blade radii from the rotor hub. Overall, this grid contains a total of 7533 observer points. Time histories of acoustic pressure were computed for each of these observer locations using the parallel Kirchhoff code on an SP2. These unsteady results were then animated and visualized on an SGI graphics workstation.

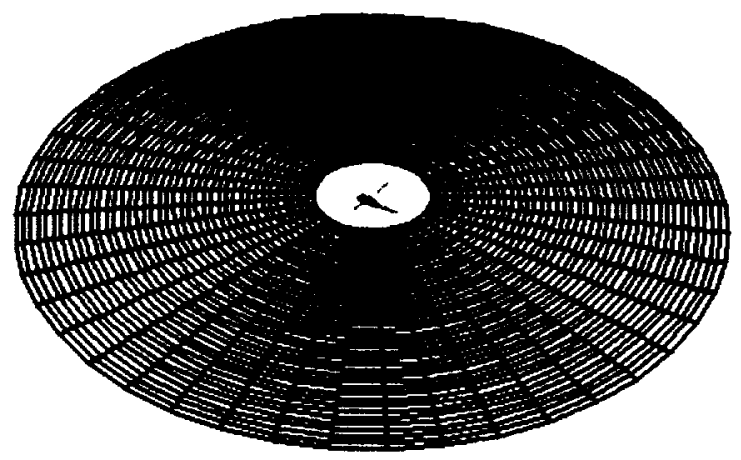

Figure 2: Far-field grid of observer points for the Kirchhoff acoustics simulation.

The animated results give a clear picture of the unsteady propagation of acoustic signals in the far field as will be shown later. This approach has the advantage that the entire field can be viewed at once. It conveys much more information than what can be seen from a handful of far-field experimental microphones. Another benefit from the Kirchhoff animations is that they can cover large regions of the acoustic far-field with high accuracy. Windtunnel geometric constraints typically limit experimental microphone placement to a region that is less than three or four radii from the rotor blades. These microphone locations may be too close to accurately represent the acoustic far-field propagation. There is no restriction however, on the locations of the far-field observer points in the computer simulations.

\section{AUDIO POSTPROCESSING}

Time-dependent CFD/Kirchhoff rotorcraft acoustics simulations are well-suited for audio playback. The CFD/Kirchhoff integration provides pressure data at discrete locations in the far-field such as those shown in Fig. 2. Audio playback is obtained by using the Stereophonic Acoustics Software Library that has been recently developed at NASA Ames Research Center. This software package allows the user to interactively select individual grid points where pressure data can be played over headphones or stereo speakers connected to an SGI Indigo computer. The digital pressures for the entire field are scanned for maximum and minimum amplitudes and then scaled for 16-bit stereo sound. The user can add a slight phase shift between the signals for each ear for added realism. This phase shift simulates the inter-aural time delay that conveys spatialized acoustic perceptions to the human brain.

Sample computed results for the OLS rotor cases in Ref. [20] have been processed for the audio playback described above. Although we cannot effectively convey the audio results in this paper, they are quite realistic. This is particularly true when played back at high volume!

\section{IDENTIFICATION OF NOISE SOURCES}

Successful design of low-noise rotors requires a connection between far-field noise predictions and the sources of noise at the rotor disk. This is particularly true for BVI cases where the highest noise levels may originate from only one or two localized blade-vortex interactions. A relatively small change in blade trajectory may locally increase the miss distance for a BVI event and drastically reduce the far-field noise. The main design issues are where and how to modify these blade trajectories.

The nonrotating Kirchhoff formulation can be modified to provide information on the locations of the main sources of rotor noise. We accomplish this by computing the integrand in Eq. (1) at each node on the Kirchhoff surface at a particular observer location and observer time when the noiee level is high. Recall that 
these integrands are all evaluated at different retarded times. A contour plot is then constructed from these nodal integrand values. This contour plot shows the relative contributions from each surface point to the farfield acoustic pressure. Since the Kirchhoff surface is only one or two chordlengths away from the rotor disk, these contour plots provide an excellent view of the locations of the noise sources near the rotor blades. When paired with some knowledge of the rotor tip-vortex locations, a designer can identify the rotor BVI events that produce the far-field noise. Modification of the blade trajectories in these regions has a high potential for noise reduction in the far field.

We use the term, "Kirchhoff-surface acoustic tracebacks," to describe this noise-source identification technique. It was first used in Ref. [11] for cases with HSI noise. Applications of the technique for BVI noise are described later in this paper.

\section{RESULTS: High-Speed Impulsive Noise}

The acoustic analysis methods described above have been applied to the OLS rotor blade cases that were experimentally-tested by Schmitz et al. [20]. In this experiment, acoustic signals were recorded at several far-field locations from a $1 / 7$ scale model of the Army's AH-1 two-bladed helicopter main rotor. The test condition for this paper has a hover-tip Mach number of 0.665 , and an advance ratio of 0.258 . This yields a maximum advancing-tip Mach number of 0.837 and significant transonic flow on the advancing side of the rotor disk. This case is modeled as a nonlifting rotor blade in order to simplify the CFD analysis. Lifting rotor calculations require fine grids to capture the rotor wake systems. This is not necessary in this case since rotor lift has little effect on HSI noise in the plane of the rotor blades [20]. The Kirchhoff surface is located approximately 1.5 chords from the rotor blade in all directions.

Reference [7] describes this case in more detail and gives comparisons of predicted acoustic pressures using the CFD/Kirchhoff method to the experimental microphone data at several locations in the far-field. Figure 3 shows one of these comparisons for a microphone located in the plane of the rotor and 3.44 rotor radii directly upwind from the rotor hub. The comparison shows excellent agreement between the prediction and the experimental data. Reference [7] shows similar results for the other microphone locations.

The observer grid in Fig. 2 is used for computing the far-field acoustic pressures in the plane of the rotor blades. A relatively fine $(81 \times 93)$ grid of far-field observers is used since the propagating acoustic waves are highly impulsive. The use of coarse grids causes interpolation errors when the acoustic waves are not alimned

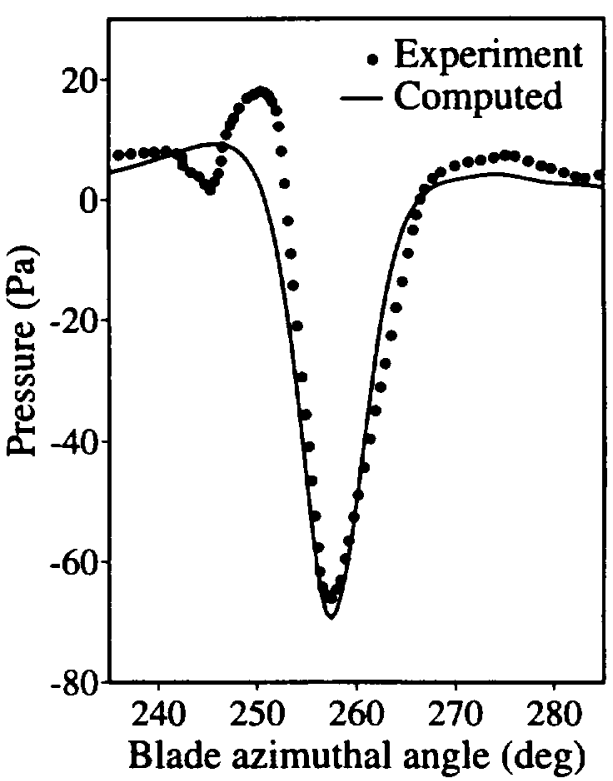

Figure 3: Comparison of predicted and experimental acoustic pressure data for the OLS rotor blade.

with the far-field observer grid. The TURNS code for this case required approximately $2.5 \mathrm{C}-90 \mathrm{CPU}$ hours to complete the unsteady solution for one blade revolution on a $(135 \times 50 \times 35)$ grid. The CPU requirement for interpolations onto the Kirchhoff surface is negligible. The nonrotating Kirchhoff surface consists of 86,400 mesh points and the temporal resolution for the observer locations corresponds to one degree of blade azimuthal angle. This means that $180 \mathrm{Kirchhoff}$ integrations are required at each of 7533 observer locations to obtain the predictions for one rotor cycle.

Figure 4 shows a snapshot from an animation of the far-field acoustic pressure contours. The time corresponds to rotor blade positions of $90^{\circ}$ and $270^{\circ}$ azimuthal angle. These pressure values are scaled by the distance from the rotor hub, which helps to show the directivity of the noise in the far field. The highest scaled acoustic amplitude occurs along the direction of flight, directly head of the rotor blade and slightly to the advancing side. The experimental data also shows this same behavior.

The animated results give a clear picture of the unsteady propagation of acoustic signals in the far field. The advantage of this approach is that the entire field can be viewed at once. This conveys much more information than that obtained from a handful of experimental microphones. Also, the far-field predictions provide acoustic information at much larger distances from the rotor hub than windtunnel experiments. The importance of this additional information is even greater for cases with BVI noise where the far-field acoustic proparation is much more complex than that shown in 


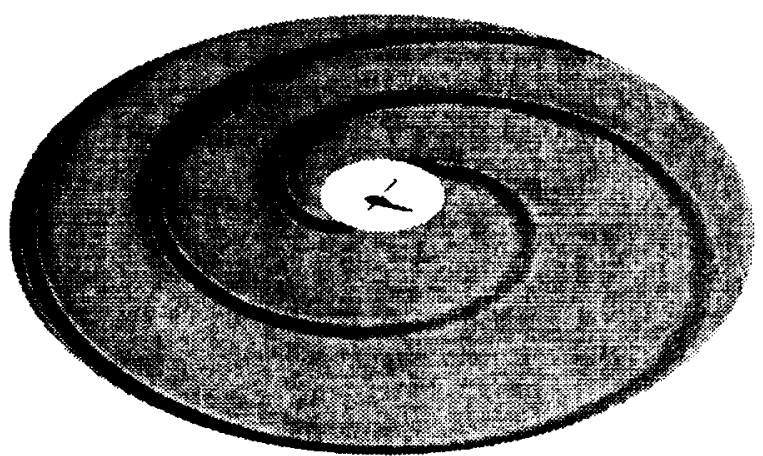

Figure 4: Acoustic pressures in the plane of the rotor for the OLS rotor blade.

Fig. 4.

The time-dependent CFD/Kirchhoff results in Fig. 4 are well-suited for audio playback. The acoustic predictions have been incorporated into an interactive computer simulation that allows the user to choose any position in the far-field relative to the spinning rotor system. The computer screen shows the helicopter fuselage and spinning rotor blades from this viewpoint. Once the viewpoint is set, the simulation reads from the stored far-field acoustic pressures to produce an audio rendering of the helicopter noise. The viewpoint can be changed to allow the user to compare the rotor noise at different locations. This type of simulation is a good model for a virtual environment that allows for rapid comparisons of helicopter noise in urban environments with a variety of flight paths for approach and landing.

\section{RESULTS: Parallel Blade-Vortex Interaction Noise}

We have also used the new acoustics analysis techniques to simulate the isolated parallel blade-vortex interaction experiment of Kitaplioglu and Caradonna [21]. In the experiment, a vortex generator is placed upstream of a rotor in a windtunnel. The vortex generator produces a vortex of known strength and trajectory. This vortex interacts with the rotor blades to produce rapid changes in lift that characterize the BVI event. The rectangular rotor blade has an aspect ratio of 7.125 and a NACA 0012 airfoil section. Figure 5 shows a schematic of the experimental and computational setup. As shown in the figure, an experimental microphone array is located 20 chords from the rotor hub at the $270^{\circ}$ azimuthal angle position on the retreating side of the rotor disk. Positive azimuthal angles are defined in the counterclockwise direction with $0^{\circ}$ being furthest away from the oncoming freestream velocity.

The computed test case is the same as that given in Ref. [7]. The TURNS code models the vortex analytically as described in Refo. $[22,23]$ and computes the

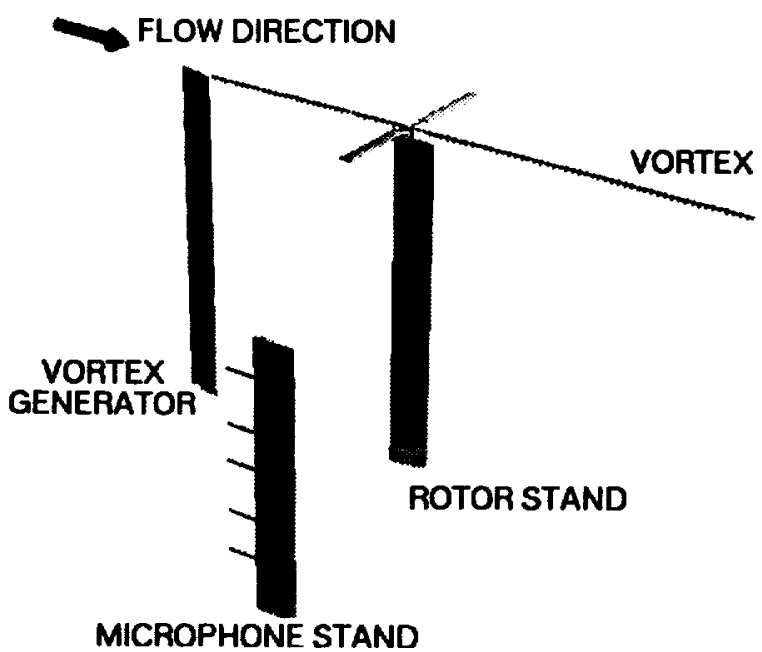

Figure 5: Experimental and computational setup for the parallel blade-vortex interaction.

surrounding flowfield to satisfy the conservation equations. The analytic vortex model simplifies the analysis since the vortex cannot diffuse from numerical dissipation in the CFD method. Computation times and grid sizes for this BVI case are essentially the same as those for the HSI case described earlier. Numerical experiments showed that timesteps equal to $0.25^{\circ}$ of rotor azimuthal angle with three Newton subiterations per timestep give far-field noise results that are independent of the grid size and choice of timestep.

The test case has a hover-tip Mach number of 0.713 , and an advance ratio of 0.197 . The vortex has a strength of $1.05 V_{\infty} c$, where $V_{\infty}$ is the tunnel freestream velocity and $c$ is the rotor chordlength. The sign of the vorticity is opposite to the direction of the freestream velocity and the vortex passes through the the rotor hub one quarter chord below the rotor disk. The Kirchhoff surface for this case is approximately 1.5 chords away from the rotor blades in all directions and numerical studies in Ref. [7] indicate that the computed noise is grid-independent at this Kirchhoff-surface location.

Figure 6 compares computed and experimental pressure values for two of the microphone locations. Microphones 2 and 5 are located 12.49 and 21.45 chords below the plane of the rotor, respectively. Additional comparisons between computation and experiment are presented in Ref. [7]. The computations and experimental data show excellent agreement for the positive part of BVI signal. However, the computations also show a negative signal that is not present in the experimental data.

Three different planar Kirchhoff meshes were used to animate the resulting far-field noise propagation. Each mesh contained 7533 points and covered a region extending from 2 to 12 blade radii similar to that shown 


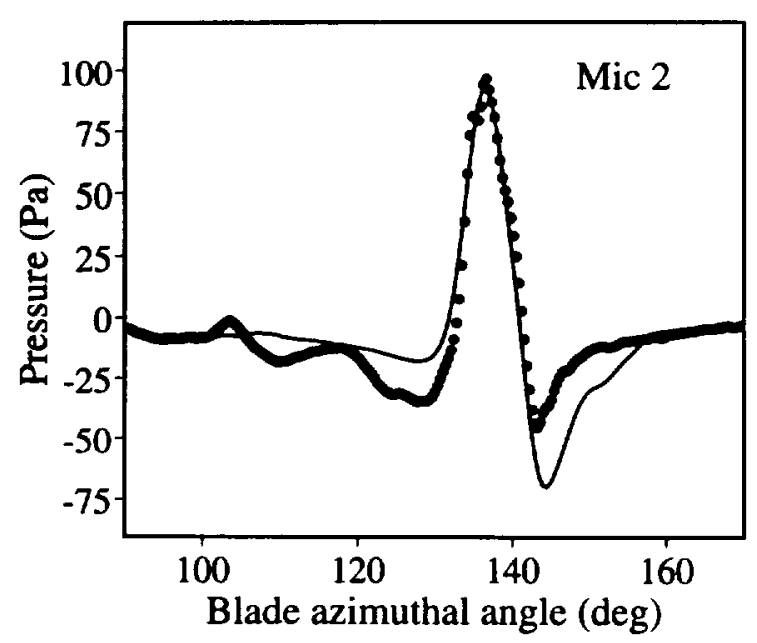

- Experiment

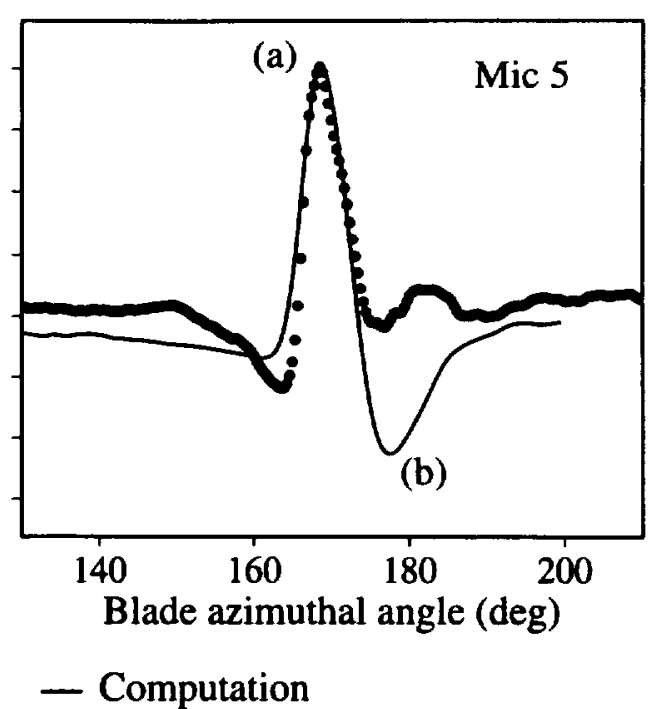

- Computation

Figure 6: Parallel BVI comparison of computed and experimental acoustic pressures.

in Fig. 2. One of the meshes is located in the plane of the rotor blades and the other two are 21.45 and 10.725 chords below the rotor plane.

Figure 7 shows a close-up of the acoustic pressure contours 21.45 chords below the rotor disk near the experimental microphone stand. Three separate acoustic waves are visible in this figure. First, the far-left side of the figure shows a wave that propagates upstream from the rotor hub. This represents a region of negative pressure and originates on the advancing side of the rotor disk near a blade azimuthal angle of $90^{\circ}$. The calculation shows quite a bit of transonic flow in this region and the resulting acoustic signal propagates ahead of the rotor as HSI noise.

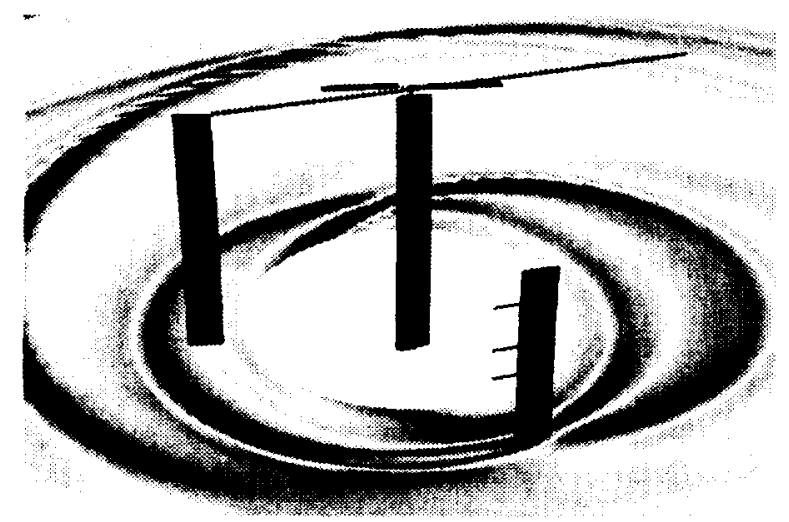

Figure 7: Closeup of acoustic propagation for a parallel BVI at 21.45 chords below the rotor disk.

The other two pressure waves are circular with their centers offset by approximately one rotor diameter. The circular wave on the left has a positive pressure and is produced by the BVI when the blade is located at $180^{\circ}$ azimuthal angle. The second wave has a nega- tive pressure and results from the BVI at $0^{\circ}$ azimuthal angle. The BVI acoustic waves propagate in a dipole pattern, which explains why they intersect the observer plane in a circle.

Note that the two circular BVI waves intersect near the location of the experimental microphones on the retreating side of the rotor disk. The negative wave from the $0^{\circ}$ BVI passes over the microphones slightly behind the positive wave from the $180^{\circ} \mathrm{BVI}$. This effect is reflected in Fig. 6, where the computed microphone pressure shows a positive peak, (a), followed by a negative peak, (b). Most likely, the vortex in the experiment dissipates considerably when it hits the rotor hub and the BVI at the $0^{\circ}$ location is not nearly as strong as the one at $180^{\circ}$. The computations assume that the vortex strength and core-size are undisturbed as it passes through the rotor hub, and that the vortices are identical at the $0^{\circ}$ and $180^{\circ}$ BVI locations. This assumption of constant vortex strength in the prediction explains the discrepancies between experimental and computational results in Fig. 6.

We can confirm this explanation by performing tracebacks to identify the sources of noise on the nonrotating Kirchhoff surface. Figure 6 marks the positive and negative peak pressures for microphone 5 with the letters (a) and (b). The contributions from the Kirchhoff surface to these acoustic pressure peaks are shown in Fig. 8. The highest pressure contours in Fig. 8a are located near the $180^{\circ}$ BVI location. The lowest pressure contours in Fig. $8 \mathrm{~b}$ are located near the $0^{\circ}$ BVI location. The resulting predicted acoustic pressures at microphone 5 show contributions from both of these BVI events. This case illustrates why it is important to include the contributions from all of the rotor blades when simulating general rotor BVI cases. 

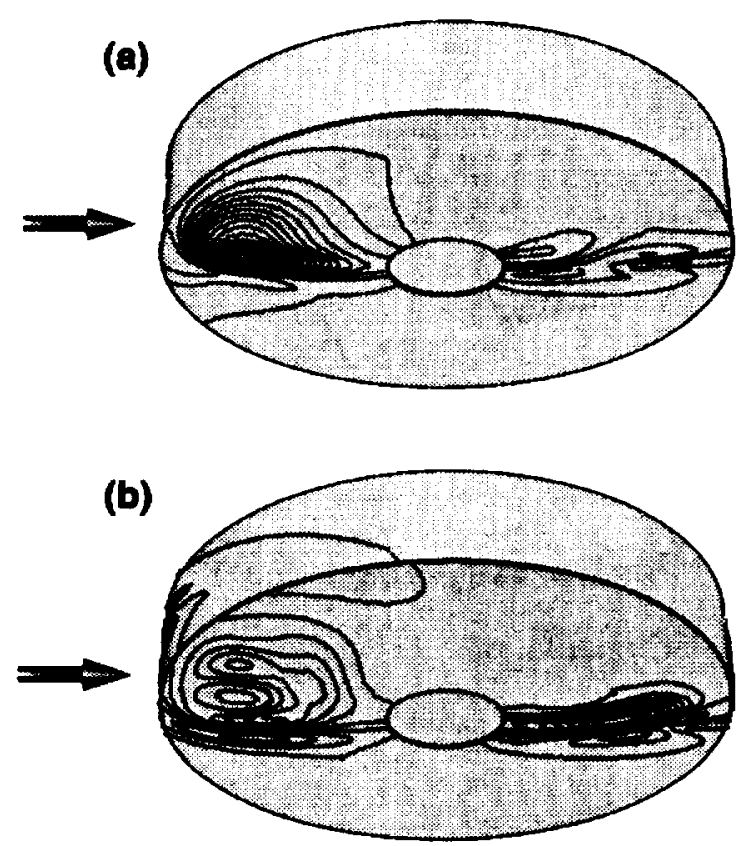

Figure 8: Acoustic tracebacks on the Kirchhoff surface for the computed positive, (a), and negative, (b), pressure peaks at microphone 5 . These views show the side and the bottom of the Kirchhoff surface.

Figure 9 shows a far-field view of the propagating acoustic waves in the same plane as shown in Fig. 7. The two BVI waves propagate to the retreating and advancing sides of the rotor disk while the HSI wave propagates upstream and dominates the acoustic signal in the far-field. It is clear from this figure that the computational results give a much more complete picture of the acoustic far field than the experimental data.

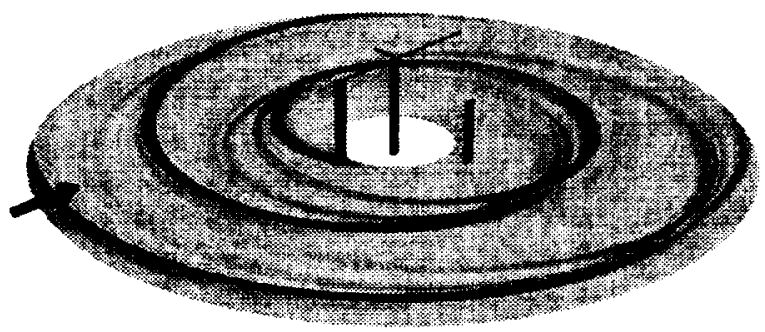

Figure 9: Far-field view of parallel BVI acoustic propagation 21.45 chords below the rotor disk.

Figure 10 shows a snapshot of the computed parallel BVI acoustic pressures in the plane of the rotor. Since the BVI acoustic waves propagate in dipole patterns, they are not visible here. HSI waves typically propagate in a monopole pattern and these dominate the in-plane rotor noise. The highest amplitude acous-

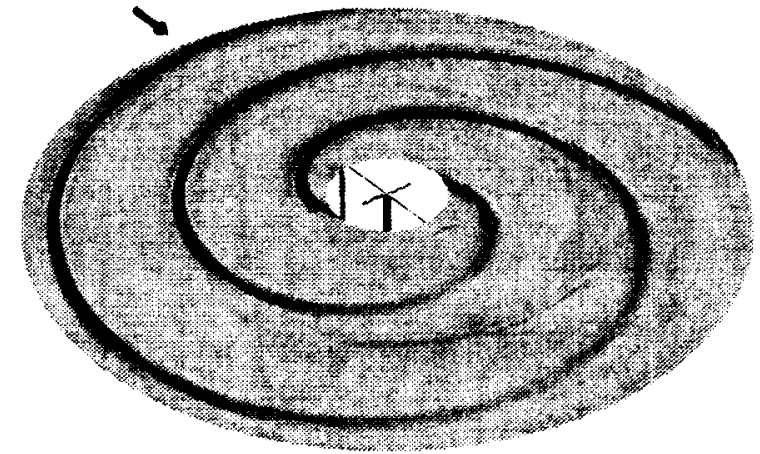

Figure 10: Parallel BVI acoustic propagation in the plane of the rotor blades.

tic signals propagate upstream from the rotor hub. All far-field acoustic pressure contours in this paper are scaled by the distance from the rotor hub.

\section{RESULTS: Oblique Blade-Vortex Interaction Noise}

In addition to parallel blade-vortex interactions, a more common scenario occurs when the vortex passes beneath the rotor blades at an oblique angle. We can simulate an idealized oblique interaction by moving the vortex generator away from the centerline of the rotor hub. Figure 11 shows such a case where the vortex has moved 2.13 chords toward the advancing side of the rotor disk. Caradonna et al. [24] have tested this configuration in the Army AFDD $7 \times 10$-foot windtunnel at Ames Research Center and recorded unsteady blade surface pressures. Far-field acoustic measurements were not recorded however. Numerical simulations of this idealized oblique BVI were performed in Ref. [24] with a full-potential CFD code and by Srinivasan and McCroskey [25] with an early version of the TURNS code.

The test conditions for this case have a hover-tip Mach number of 0.763 , and an advance ratio of 0.197 . The vortex has a strength of $1.218 V_{\infty} c$ and the sign of the vorticity is aligned with the freestream velocity. The vortex is located 0.25 chords below the plane of the rotor and the outer tip of the rotor blade passes over the vortex on the advancing side at $17^{\circ}$ and at $163^{\circ}$ blade azimuthal angle. Large regions of transonic flow occur near the tips of the rotor blades between $70^{\circ}$ and $170^{\circ}$ azimuthal angle. This means that the BVI event in the second quadrant of rotor motion is highly nonlinear and unsteady.

The TURNS code was run in the Euler mode for this simulation using the same computational grid that was used for the parallel interaction case. Because of the highly unsteady flow in the second quadrant of rotor motion, the timestep was reduced to an equivalent of 0.125 degrees azimuthal angle with five Newton subiterations at each timestep. Numerical experiments showed 


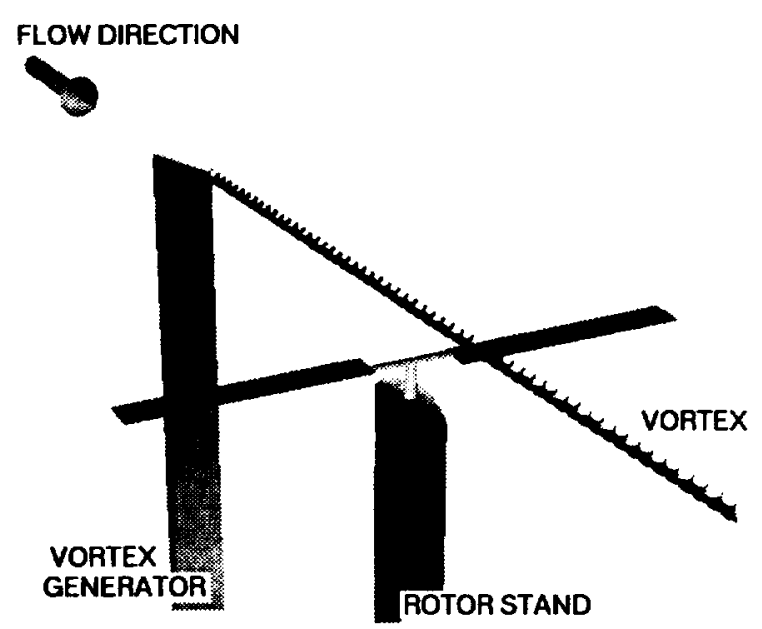

Figure 11: Experimental and computational setup for an idealized oblique BVI.

that these choices yielded solutions that were independent of the choice of timestep. Due to the strong transonic flow in this case, the Kirchhoff surface was located at a distance of two chords from the rotor blades in all directions.

Computed results for blade surface pressures are similar to those computed by Caradonna et al. [24] and Srinivasan and McCroskey [25] for the same test case. Minor differences appear near $165^{\circ}$ azimuthal angle, where the highest transonic and unsteady effects are present. Differences between Ref. [25] and the current calculations are most likely due to the differences in the computational grids. Srinivasan and McCroskey used a $21 \times 101 \times 15 \mathrm{O}-\mathrm{O}$ mesh with the outer boundaries located 8 chords from the rotor in all directions. The present calculations used a $151 \times 40 \times 50 \mathrm{C}-\mathrm{H}$ mesh with outer boundaries located approximately 12 chords away from the rotor blade in all directions.

Figure 12 shows a snapshot of the computed pressure contours at 21.45 chords below the rotor disk. Three distinct acoustic waves can be seen in a region that is between 3 and 7 rotor radii upstream of the rotor hub. All three of these waves propagate upstream and each is caused by a different event on the rotor blades. The first of these three waves (as seen by a stationary observer) has a positive pressure and is the result of the downstream BVI event near $17^{\circ}$ blade azimuthal angle. The second wave has a negative pressure and is caused by the high-speed impulsive noise the emanates from the rotor blade tip in the second quadrant of ro tor motion. The third wave also has a negative pressure and comes from the BVI event near $163^{\circ}$ blade azimuthal angle. The origins of these three acoustic waves are fairly clear from the complete animation of the computed acoustic pressures. These noise sources can be confirmed by examining the acoustic tracebacks

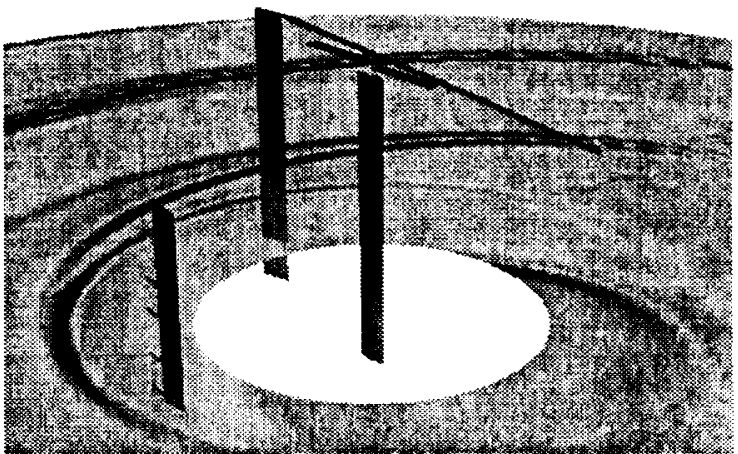

Figure 12: Acoustic propagation for the oblique BVI.

to the Kirchhoff surface for an observer that is located upstream of the rotor hub.

Figure 13 shows three acoustic tracebacks for an observer who is located 30.28 chords upstream from the rotor hub and 21.45 chords below the rotor disk. The pressure vs. azimuthal angle plot shows that the observer hears three distinct pressure peaks and these are labeled (a),(b), and (c) in the figure. The first is a positive pressure, and the source of this noise is shown on the Kirchhoff surface in Fig. 13a. The main contribution to the pressure at point (a) comes from the lower part of the Kirchhoff surface, near the BVI event at $17^{\circ}$ blade azimuthal angle. The second pressure peak occurs at point (b), and almost all of the contributions here come from the advancing side of the Kirchhoff surface as shown in Fig. 13b. This negative pressure region is caused by HSI noise on the advancing side, which radiates from the rotor blades in a monopole pattern. Finally, the third pressure peak occurs at point (c), and the sources of this noise are shown in Fig. 13c. This figure shows both positive and negative pressures on the side of the Kirchhoff surface in the second quadrant. These disturbances result from the delocalized shock wave and their net contribution to the far-field observer pressure is small. The dominant contribution in Fig. 13c to the pressure peak at point (c) comes from the lower section of the Kirchhoff surface close to the BVI event near $163^{\circ}$ blade azimuthal angle. The acoustic tracebacks confirm the earlier explanation for the origins of the three acoustic waves shown in Fig. 12

\section{SUMMARY AND CONCLUSIONS}

This paper describes several new methods to predict and analyze rotorcraft noise. Individually, these methods are: 1) CFD/Kirchhoff far-field noise predictions, 2) parallel computer implementation of the Kirchhoff integrations, 3) audio and visual rendering of the computed acoustic predictions over large far-field regions, and 4) acoustic tracebacks to the Kirchhoff surface to pinpoint the sources of the rotor noise. When taken together, these methods exploit the power of new 

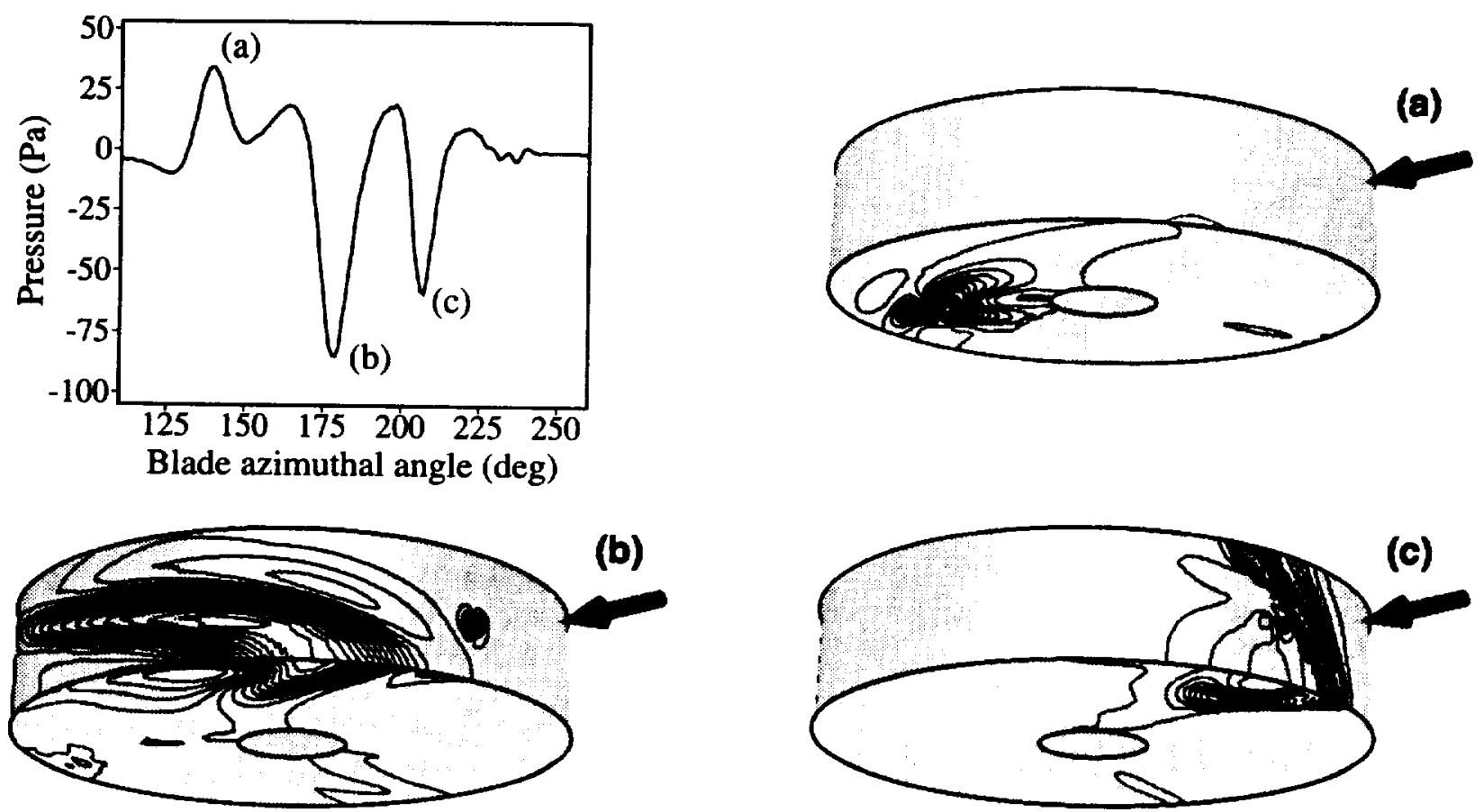

Figure 13: Acoustic tracebacks on the Kirchhoff surface for an oblique BVI. The observer location is 30.28 chords upstream of the rotor hub and 21.45 chords below the plane of the rotor. Viewpoint shows bottom and side of the Kirchhoff surfaces.

computer technologies and offer the potential to significantly improve our prediction capabilities and understanding of rotorcraft noise.

These four prediction and analysis methods convey much more information about the propagation of rotorcraft noise than can be obtained from typical experiments. The visual rendering shows details of the acoustic propagation over large regions in the far field, and the acoustic tracebacks to the Kirchhoff surface help to identify the noise sources at the rotor disk. The computed results give an accurate picture of both the far-field noise and its origins on the rotor blades.

The current CFD/Kirchhoff methods provide accurate simulations for just about any type of in-plane HSI noise. This is because the rotor wake system does not play a major role in this type of noise. The CFD/Kirchhoff analysis would be a good candidate for a blade-design optimization study to reduce HSI noise. General cases with BVI noise are more difficult because the CFD codes have trouble capturing the rotor vortex wake systems without excessive numerical dissipation. This is an active area of research and we expect to see dramatic improvements in CFD rotor-wake capturing within the next five years. The Kirchhoff integration method in this paper is compatible with any rotarywing CFD code, so that future improvements in CFD wake capturing should directly result in improved BVI noise predictions.

\section{ACKNOWLEDGMENTS}

The authors would like to thank Vee Hirsch for her work to produce the computer-animated video that accompanies this paper. We would also like to thank G.R. Srinivasan for his help with the TURNS code for the oblique BVI case. Both of these individuals work for Sterling Software at the NASA Ames Research Center.

\section{REFERENCES}

[1] Xue, Y., and Lyrintzis, A. S., "Rotating Kirchhoff Method for Three-Dimensional Transonic BladeVortex Interaction Hover Noise," AIAA Journal, Vol. 32, No. 7, July 1994, pp. 1350-1359.

[2] Lyrintzis, A. S., Kilaras, M. S., and Xue, Y., "Transonic 3-D BVI Noise Using a Rotating Kirchhoff Formulation for Advancing Rotors," 50th AHS Annual Forum, Washington, DC, May 1994.

[3] Baeder, J. D., Gallman, J. M., and Yu, Y. H., “A Computational Study of the Aeroacoustics of Rotors in Hover," 49th AHS Annual Forum, St. Louis, MO, May 1993.

[4] Strawn, R. C., Biswas, R., and Garceau, M., "Unstructured Adaptive Mesh Computations of Rotorcraft High-Speed Impulsive Noise," Journal of Aircraft, Vol.-32, No. 4, July-Aug. 1995, pp. 754-760.

[5] Strawn, R. C., and Biswas, R., "Numerical Simulations of Helicopter Aerodynamics and Acoustics," 
Journal of Computational and Applied Mathematics, to appear.

[6] Strawn, R. C., and Biswas, R., "Computation of Helicopter Rotor Noise in Forward Flight," Journal of the American Helicopter Society, Vol. 40, No. 3, July 1995 , pp. $66-72$.

[7] Strawn, R. C., Biswas, R., and Lyrintzis, A. S., "Helicopter Noise Predictions Using Kirchhoff Methods," Journal of Computational Acoustics, Vol. 4, No. 3, Sep. 1996.

[8] Lyrintzis, A. S., "Review: The Use of Kirchhoff's Method in Computational Aeroacoustics," Journal of Fluids Engineering, Vol. 116, Dec. 1994, pp. 665675.

[9] Polacsek, C., and Costes, M., "Rotor Aeroacoustics at High-Speed Forward Flight using a Coupled Full Potential/Kirchhoff Method," 21st European Rotorcraft Forum, St. Petersburg, Russia, Aug. 1995.

[10] Lyrintzis, A. S., Koutsavdis, E. K., Berizin, C. R., Visintainer, J. A., and Pollack, M. J., "Kirchhoff Acoustic Methodology Validation and Implementation in the Tiltrotor Aeroacoustic Codes (TRAC)," 2nd AHS Aeromechanics Specialists' Conference, Bridgeport, CT, Oct. 1995.

[11] Duque, E. P. N., Strawn, R. C., Ahmad, J., and Biswas, R., "An Overset Grid NavierStokes/Kirchhoff-Surface Method for Rotorcraft Aeroacoustic Predictions," 34th AIAA Aerospace Sciences Meeting, Reno, NV, Jan. 1996, Paper 960152.

[12] Lowson, M., "Directionality of Helicopter BVI Noise," 21st European Rotorcraft Forum, St. Petersburg, Russia, Aug. 1995.

[13] Sim, B. W-C., George, A. R., and Yen, S. J., "BladeVortex Interaction Directivity Studies Using Trace Mach Number," 2nd AHS Aeromechanics Specialists' Conference, Bridgeport, CT, Oct. 1995.

[14] Srinivasan, G. R., Baeder, J. D., Obayashi, S., and McCroskey, W. J., "Flowfield of a Lifting Rotor in Hover: A Navier-Stokes Simulation," AIAA Journal, Vol. 30, No. 10, Oct. 1992, pp. 2371-2378.

[15] Srinivasan, G. R., and Baeder, J. D., "TURNS: A Free-Wake Euler/Navier-Stokes Numerical Method for Helicopter Rotors," AIAA Journal, Vol. 31, No. 5, May 1993, pp. 959-962.

[16] Farassat, F., and Myers, M. K., "Extension of Kirchhoff's Formula to Radiation from Moving Surfaces," Journal of Sound and Vibration, Vol. 123, No. 3, 1988, pp. 451-460.

[17] Myers, M. K., and Hausmann, J. S., "On the Application of the Kirchhoff Formula for Moving Surfaces," Journal of Sound and Vibration, Vol. 139, 1990, pp. 174-178.
[18] Meakin, R., "Moving-Body Overset Grid Methods for Complete Aircraft Tiltrotor Simulations," 11th AIAA Computational Fluid Dynamics Conference, Orlando, FL, July 1993, Paper 93-3350.

[19] Suhs, N. E., and Tramel, R. E., "PEGSUS 4.0 Users Manual," Arnold Engineering Development Center, AEDC-TR-91-8, June 1991.

[20] Schmitz, F. H., Boxwell, D. A., Splettstoesser, W. R., and Schultz, K. J., "Model-Rotor HighSpeed Impulsive Noise: Full-Scale Comparisons and Parametric Variations," Vertica, Vol 8, No. 4, 1984, pp. 395-422.

[21] Kitaplioglu, C., and Caradonna, F. X., "A Study of Blade-Vortex Interaction Aeroacoustics Utilizing an Independently-Generated Vortex," AGARD Fluid Dynamics Panel Symposium on Aerodynamics and Aeroacoustics of Rotorcraft, Berlin, Germany, Oct. 1994.

[22] Srinivasan, G. R., "A Free-Wake Euler and NavierStokes CFD Method and its Application to Helicopter Rotors Including Dynamic Stall," JAI Associates Inc., TR 93-01, Nov. 1993.

[23] Baeder, J. D., and Srinivasan, G. R., "Computational Aeroacoustic Study of Blade-Vortex Interaction Noise," AHS Aeromechanics Specialists' Meeting, San Francisco, CA, Jan. 1994.

[24] Caradonna, F. X., Strawn, R. C., and Bridgeman, J. O., "An Experimental and Computational Study of Rotor-Vortex Interactions," Vertica, Vol. 12, No. 4, 1988, pp. 315-327.

[25] Srinivasan, G. R., and McCroskey, W. J., "Euler Calculations of Unsteady Interaction of Advancing Rotor with a Line Vortex," AIAA Journal, Vol. 31, No. 9, Sep. 1993. 


